\title{
Expert*innendissens und das reversible Verfahren der Suche nach einem Endlagerstandort für hochradioaktive Abfälle
}

Saleem Chaudry, Roman Seidl

\section{Zusammenfassung}

Durch ein gesetzlich geregeltes Verfahren soll derzeit der Standort gefunden werden, der die bestmögliche Sicherheit zur Entsorgung der hochradioaktiven Abfälle der Bundesrepublik Deutschland bietet. Das Standortauswahlverfahren soll lernend, selbsthinterfragend, und damit reversibel sein. Das Feld ist von vielfältigem Expertenwissen und -handeln geprägt. Änderungen im reversibel gestalteten Verfahrensablaufdurch Erkenntnisgewinn oder Neubewertung bestehenden Wissens sind zu erwarten. Dissens zwischen Experten kann in diesem Zusammenhang eine wesentliche Rolle spielen. Um Handlungsempfehlungen für reversible Verfahren im Allgemeinen und das Standortauswahlverfahren im Besonderen zu formulieren, müssen die Mechanismen und Wirkungen von Expertendissensen besser verstanden werden. So kann Dissens über den wissenschaftlichen Status-Quo fruchtbar sein, da er Unsicherheiten im Wissen aufdeckt und neue Forschung ermöglicht. Andererseits kann er bei Bürgern und Entscheidern zu Verunsicherung führen. Expertendissens kann darüber hinaus instrumentell eingesetzt werden, um beispielsweise politische Ziele zu verfolgen.

Im Hinblick auf die Reversibilität des Standortauswahlverfahrens soll dieser Beitrag klären helfen, ob und wie Expertendissens auf Entscheidungsprozesse Wirkung entfalten kann. Dazu werden eine Definition von Expertendissens basierend auf Sekundärliteratur erarbeitet sowie das Konzept der Reversibilität näher beschrieben. Das im StandAG festgehaltene Reversibilitätsverständnis wird anderen Ansätzen aus verschiedenen Disziplinen gegenübergestellt. Anhand historischer Fälle von Expertendissens im Rahmen von Endlagerprojekten wird eine Typologie entwickelt und untersucht, ob und wie diese historischen Dissense Wirkung im jeweiligen Kontext entfaltet haben. Zudem wird geprüft, welche Lehren daraus für das Standortauswahlverfahren gezogen werden können. 


\section{Einführung}

Durch ein gesetzlich geregeltes Verfahren soll derzeit der Standort gefunden werden, der die bestmögliche Sicherheit zur Entsorgung der hochradioaktiven Abfälle der Bundesrepublik Deutschland bietet. Damit wird auch auf frühere, erfolglose Versuche reagiert, einen Endlagerstandort zu identifizieren, der zudem gesellschaftlich und wissenschaftlich akzeptabel ist. Dieses mit der Verabschiedung des Standortauswahlgesetzes (StandAG) 2013 initiierte neue Verfahren folgt unter anderem dem Anspruch, lernend, selbsthinterfragend, und damit reversibel zu sein (zu Reversibilität im Standortauswahlgesetz siehe unten; zum Reversibilitätsbegriff siehe auch den Beitrag von Mbah et al. »Reversibilität im Kontext der Entsorgung hochradioaktiver Abfälle« in diesem Band). Reversibilität wird dabei an festgelegte Verfahrensschritte gebunden. Verzögerung oder Anhalten eines Verfahrens oder gar die Zurücknahme oder Änderung bereits vollzogener Verfahrensschritte erfordern Entscheidungen, die wiederum guter Gründe bedürfen.

Ein möglicher Grund für Änderungen am Verfahrensablauf kann sein, dass sich neue Erkenntnisse, neue Bewertungen bekannten Wissens oder veränderte Prioritäten bei der Entscheidungsfindung ergeben. Jedenfalls müssen Informationen zu geänderten (sozialen, politischen, ökonomischen) Rahmenbedingungen vorliegen, die die jeweils aktuelle Planung in Frage stellen. Expert*innendissens kann besonders in einem Feld, das so sehr von vielfältigem Expert*innenwissen und -handeln geprägt ist wie die Entsorgung radioaktiver Abfälle (Smeddinck et al. 2020), eine herausragende Rolle spielen.

Ein reversibles Verfahren transparent durchzuführen, stellt hohe Ansprüche an alle Beteiligten, insbesondere aber an die Entscheidungsträger (Drögemüller 2018; Aparicio 2010). Ein Verständnis der Mechanismen und Wirkungen von Expert*innendissensen kann dabei helfen, Handlungsempfehlungen für reversible Verfahren im Allgemeinen und das Standortauswahlverfahren im Besonderen zu formulieren.

Der Gesetzgeber, aber auch die Öffentlichkeit, erwarten, dass das Verfahren wissenschaftlich abgestützt zum Standort mit der größtmöglichen Sicherheit führt. Dissens über den wissenschaftlichen Status-Quo kann Unsicherheiten im Wissen aufdecken und neue Forschung ermöglichen, jedoch bei Bürger*innen und Entscheider*innen auch zu Verunsicherung führen. Andererseits können Transparenz und Authentizität auch zu mehr Vertrauen in das Verfahren führen, wenn Unsicherheiten richtig kommuniziert werden. Es besteht weiterhin die Möglichkeit, dass Expert*innendissens instrumentell eingesetzt wird, um politische Ziele zu verfolgen.

Es besteht daher im Hinblick auf die Reversibilität des Standortauswahlverfahrens Klärungsbedarf, ob und wie Expert*innendissens Wirkung auf Entscheidungsprozesse entfalten kann. Wird etwa bei unterschiedlicher Interpretation von Fakten abgewartet, bis Streitfragen durch zusätzliche Forschung beziehungsweise Abklärung soweit beantwortet werden können, dass Entscheidungen akzeptabel getroffen werden können? Kurz, sind Rücksprünge und Änderungen des gesetzlichen oder politischen Pfades (siehe auch den Beitrag von Losada »Pfadabhängigkeiten in der Endlagerpolitik« in diesem Band) innerhalb des Verfahrens möglich? Wie können zu erwartende Expert*innendissense im Verfahrensverlauf berücksichtigt werden? 
Im vorliegenden Beitrag wird zunächst eine Definition von Expert*innendissens basierend auf Sekundärliteratur erarbeitet (Abschnitt 1). Im Anschluss wird das Konzept der Reversibilität näher beschrieben und die Definition aus dem StandAG anderen Ansätzen aus verschiedenen Disziplinen gegenübergestellt (Abschnitt 2). In Abschnitt 3 wird anhand historischer Fälle von Expert"innendissens im Rahmen von Endlagerprojekten eine Typologie erstellt und der Frage nachgegangen, ob und wie diese Dissense Wirkung im jeweiligen Kontext entfaltet haben. Zudem wird geprüft, welche Lehren daraus für das Standortauswahlverfahren zu ziehen wären.

\section{Expert*innendissens}

Zunächst stellt sich die Frage, wer als Experte oder Expertin gilt. Meist scheint die Bezeichnung Expert*in eine Zuschreibung zu sein, sei es durch die Medien oder die Politik, vor Gericht (Gutachten) oder in Diskussionen mit Bürgerinitiativen (siehe hier$\mathrm{zu}$ auch den Beitrag von Themann et al. »Arenen zur Austragung von Dissensen in der Endlagerpolitik « in diesem Band). Eine Person sgilt« als Expert*in zu einem bestimmten Thema und wird daher als solche adressiert und zitiert. Aber weshalb genau? Ein Element, um als Expert*in zu gelten, ist das Wissen auf einem spezifischen Themengebiet. Hitzler et al. (1994: 6) sprechen von »sozialer Etikettierung [...] aufgrund spezieller Kompetenzansprüche und/oder Kompetenzunterstellungen«. Auch nach Böschen (2000) sind »Wissenschaftler [...] keine Expert*innen qua Profession, sie erhalten diese Rolle erst im Kontext institutioneller Entscheidungsprozesse.« Bei Böschen steht allerdings die Politik im Zentrum: »Wir müssen uns also vergegenwärtigen, dass Wissenschaftler erst durch Politik zu Expert*innen gekürt werden und dabei ganz grundlegende Bedingungen der eigenen Professionalität (wie systematischen Zweifel, oder NichtWissen) ausblenden müssen« (Böschen 2000: 48).

Die gestiegene Nachfrage nach Wissen, die Vielzahl der Wissensproduzenten und die zunehmende Unschärfe hinsichtlich wissenschaftlicher Gütekriterien führen zu Wissenskonflikten auch in Form von Expert*innenstreit. Der ist jedoch keine neue Erscheinung. Das Phänomen, dass Expert*innen unterschiedlicher Meinung sind, kennt man in der Literatur unter den Begriffen Expert*innenstreit (Schüssler 2015), Expert*innendilemma (Nennen/Garbe 2012) oder Expert*innendissens (Bogner 2010).

Dabei ist es Teil der Wissenschaftskultur (und evtl. von wissenschaftlichem Fortschritt), gerade Unsicherheit, Weiterfragen und Kritik zuzulassen oder kurz »Vermutungen und Widerlegungen« (Popper 2009) im Prozess zu gestalten. Es ist somit zunächst - wissenschaftsintern - nichts Ungewöhnliches, dass es zu bestimmten Fragen unterschiedliche Expert*innenmeinungen gibt. Es gibt unterschiedliche Disziplinen und methodische Ansätze, ja auch unterschiedliche Möglichkeiten, Fakten zu interpretieren:

»Es bleibt also viel Raum, um aufgrund derselben Daten zu unterschiedlichen Schlussfolgerungen zu gelangen. Deshalb werden Methoden in Frage gestellt und die Interpretationen von Fachkolleg*innen angezweifelt. Dieser kultivierte Streit ist geradezu ein Merkmal des wissenschaftlichen Prozesses. Konträre Ergebnisse sind nicht nur auf 
die Parteilichkeit von Expert*innen zurückzuführen, sondern liegen in der Natur der Sache.«(Siegrist 2001: 38)

Dissense können in dem Sinne produktiv wirken, wenn sie Aspekte zur Debatte stellen, die sonst nicht ausreichend sichtbar werden. In der aktuellen sogenannten »Corona-Krise« ist dies sehr deutlich geworden: (inner)wissenschaftliche Diskussionen werden öffentlich gemacht. Bürger*innen wie Entscheidungsträger*innen sind für jede Information dankbar, die vermeintlich »richtige« Entscheidungen ermöglicht. Allerdings wird dies dann problematisch, wenn - wie Böschen (2000) darstellt - in Entscheidungssituationen unterschiedliche Expert"innenmeinungen unterschiedliche Handlungen nahelegen. Im Kontext der Entsorgung hochradioaktiver Abfälle und der damit verbundenen langen Zeitperspektive werden Politiker"innen ganz besonders daran gemessen, dass sie heute Entscheidungen treffen, die sich für viele zukünftige Generationen als richtig erweisen müssen.

Dissens zwischen hinzugezogenen Expert*innen weist auf Unsicherheiten hin. Entscheidungsträger"innen müssen auch ohne Konsens in wissenschaftlichen Fragen entscheiden. Sie sind nicht angewiesen auf die Lösung von Dissensen, letztlich müssen Gewissensentscheidungen gefällt werden, wie etwa in der Stammzellenforschung (Bogner/Menz 2010).

Auch die mögliche negative Wirkung auf das Vertrauen der Bürger in die Wissenschaft ist zu bedenken: "Scientific knowledge is in many areas provisional, uncertain and incomplete. Thus, competing expert knowledge has in many instances given rise to a battle between experts and counter-experts. Corporate science has contested environmental advocacy science and vice versa. [...] The erosion of the legitimating function of science in certain domains has spurred the calls for making science more accountable and democratic« (Bäckstrand 2003, S. 30). Auch, wenn die Wirkung von Expert*innendissens nicht immer negativ sein und in einem Verlust von Glaubwürdigkeit enden muss (siehe Transparenz und Authentizität), sollten solche möglichen Dynamiken in der öffentlichen Wahrnehmung antizipiert werden.

Im Abschnitt 3 werden die Wirkungen von Expert*innendissensen an Hand dreier Fallstudien aus Endlagervorhaben empirisch untersucht, systematisiert und die Rollen der jeweiligen Expert*innen dargestellt. Bei der Befassung mit den vergangenen und aktuellen bundesdeutschen und anderen nationalen Entsorgungsprojekten wurde deutlich, dass die Zuschreibung von Expertise immer mit einer wissenschaftlichen Profession einhergeht. Die Akteur*innen der Dissense sind jeweils als Wissenschaftler"innen an Universitäten oder Forschungseinrichtungen beschäftigt, als Gutachter*innen tätig oder in anderer Weise wissenschaftlich mit dem Thema Endlagerung befasst. Laienexpertise, die in verschiedenen anderen Zusammenhängen, auch bei ökologisch relevanten Fragestellungen und in der Nachhaltigkeitsdiskussion, aktuell verstärkt gefragt ist (Feindt, Peter, H./Newig 2005; Newig et al. 2019), spielte bei der Entsorgung radioaktiver Abfälle in Deutschland in der Vergangenheit praktisch keine Rolle. Die Quellenlage zu den beiden konfliktreichen Endlagerprojekten am Standort Gorleben und in der Schachtanlage Asse II zeigt deutlich, dass der zivilgesellschaftliche Widerstand in Entscheidungssituationen, für Gutachten, Anhörungen oder gerichtliche Auseinandersetzungen auf wissenschaftliche Expertise zurückgreift. Die Opposition geht von der 
Bevölkerung am Standort oder in der Umgebung des Standorts aus und wird von Laien getragen; die fachliche Begründung dazu wird von wissenschaftlichen Akteur*innen geliefert. Eine mögliche Ursache ist die Randständigkeit der Themenkomplexe Kernenergie oder Endlagerung: Sie spielen im Alltag von Bürger*innen praktisch keine Rolle (im Gegensatz beispielsweise zu Sondermüll, mit dem man im Alltag eher zu tun hat) (Seidl et al. 2017). Sie stellen selbst im wissenschaftlichen Disziplinenkanon Spezialgebiete dar. Expertise in den damit verbundenen Fragestellungen wird, anders als bei vielen anderen umweltrelevanten Fragestellungen wie bspw. Siedlungsabfallentsorgung, Fluglärm oder Verkehrsplanung, ausschließlich durch gezieltes Studium gewonnen.

\section{Reversibilität und das Standortauswahlverfahren}

Das Verständnis des Begriffs Reversibilität im Zusammenhang der Endlagerstandortsuche ist entscheidend für die Frage, ob ein Expert*innendissens in einem reversiblen Verfahren Wirkung entfalten kann. Das Verfahren muss dazu sensitiv oder resonanzfähig sein für das Bedürfnis oder die erkannte Notwendigkeit zu Änderungen im Verlauf. Mit dem Reversibilitätsbegriff setzen sich die Autor*innen im Beitrag »Reversibilität im Kontext der Entsorgung hochradioaktiver Abfälle« (Mbah et al. in diesem Band) ausführlich auseinander.

Im Standortauswahlgesetz wird Reversibilität, explizit und implizit, über drei Projektionsflächen unterschiedlicher Größe abgebildet: zum einen über die Begriffsbestimmung, zum anderen über die Umsetzung im Rahmen der einzelnen Verfahrensschritte, und am umfangreichsten über den in $\$ 1$ des Gesetzes definierten ethischen Anspruch an das Verfahren. Den einfachsten Zugang ermöglicht die Begriffsbestimmung, nach der Reversibilität als »Möglichkeit der Umsteuerung im laufenden Verfahren zur Ermöglichung von Fehlerkorrekturen« (Deutscher Bundestag 2017) definiert wird. Damit wird Reversibilität im Zusammenhang des Gesetzes auch explizit auf die Standortauswahl beschränkt - nicht nur, weil das Standortauswahlverfahren mit der Standortentscheidung abgeschlossen und der Regelungsinhalt des StandAG abgearbeitet sein wird, sondern auch durch den expliziten Hinweis auf das laufende Verfahren. Eine sich auf das an die Standortauswahl anschließende Genehmigungsverfahren nach dem Atomgesetz (AtG) (Deutscher Bundestag 1959) erstreckende zeitliche Gültigkeit des Begriffs ist hier nicht vorgesehen.

Die zweite Projektionsfläche ist größer. Zur Zweckbestimmung des Gesetzes gehört auch die Reversibilität des Standortauswahlverfahrens »nach Maßgabe der \$\$ 12ff.» (Deutscher Bundestag 2017). Hier wird der Bezug zur Durchführung des Standortauswahlverfahrens hergestellt; es bleibt der Anwender*in überlassen, nachzuvollziehen, wo sich in den das Verfahren im Detail regelnden $\$ \$ 12-21$ der Reversibilitätsgedanke in welcher Ausprägung niedergeschlagen hat und wo andererseits vorgegebene Haltepunkte wie beispielsweise Entscheidungen nach Bundesgesetz reversibles Handeln erschweren oder begrenzen. Das gilt für die Betreibergesellschaft wie die Regulierungsbehörde, aber auch für jede/n andere/n Bürger*in, der/die sich mit dem StandAG auseinandersetzt. 
Die umfassendste Aussage zur Reversibilität trifft der Gesetzgeber im StandAG mit der Formulierung vom »partizipativen, wissenschaftsbasierten, transparenten, selbsthinterfragenden und lernenden Verfahren« (Deutscher Bundestag 2017). Auch hier gilt, dass es dem Anwender zukommt, den Anspruch umzusetzen und so mit Leben zu füllen, und allen Stakeholdern, unabhängig von ihrer jeweiligen Rolle, diese Umsetzung $\mathrm{zu}$ hinterfragen. Die Begriffe partizipativ und transparent fordern dazu auf. Die Möglichkeit einer Wirkung von Expert*innendissens in diesem Umfeld wird an erster Stelle durch das Adjektiv wissenschaftsbasiert erschlossen. Wissenschaftsbasiert kann heißen, dass wissenschaftlicher Konsens als Voraussetzung für das Fortschreiten des Verfahrens angesehen wird und Dissens zu Halt und Neubewertung oder Umsteuern führen kann. Konsens ist zwar zu fordern. Kritisch zu prüfen ist jedoch, ob ein Konsens nur oberflächlich als solcher erscheint. Ein Konsens im Sinn einer Zustimmung aller Akteur*innen kann in einem Verfahren dieser Größenordnung nicht realistisch als notwendige Bedingung gesetzt werden. Divergierende Meinungen und Erkenntnisse sind daher im Verfahren ein wichtiger Bestandteil.

Die Kommission Lagerung hochradioaktiver Abfallstoffe (Endlagerkommission) hat in ihrem Abschlussbericht (Kommission Lagerung hoch radioaktiver Abfallstoffe 2016a), der der Überarbeitung und Neufassung des StandAG $2017 \mathrm{zu}$ Grunde lag, weitergehende Formulierungen zur Reversibilität verwendet:

»Die Kommission hat aus der Vergangenheit Lehren gezogen und auf dieser Basis Zielsetzungen für den Weg zu einer sicheren Entsorgung festgelegt. Diese sind eine weitestgehende Reversibilität von Entscheidungen und die Realisierung des Verfahrens im transparenten Dialog mit der Öffentlichkeit. Das Prinzip der Reversibilität von Entscheidungen resultiert zum einen aus dem Wunsch nach Möglichkeiten der Fehlerkorrektur im Falle unerwarteter Entwicklungen, zum anderen aus dem zukunftsethischen Prinzip, zukünftigen Generationen Entscheidungsoptionen offen zu halten oder sie zu eröffnen. Die Beteiligung der Öffentlichkeit nach klaren Regeln und mit klaren Rechten ist geboten, um Vertrauen in das Verfahren zu schaffen und um in der Suche nach der Option mit der bestmöglichen Sicherheit möglichst viele Perspektiven zu berücksichtigen.«(Kommission Lagerung hoch radioaktiver Abfallstoffe 2016a: 26f.).

»Konzeptionell neu ist die an zukunftsethischen Prinzipien und dem Wunsch nach weitgehenden Möglichkeiten der Fehlerkorrektur ausgerichtete Forderung nach Reversibilität einmal getroffener Entscheidungen im Sinne eines lernenden Verfahrens, um das Ziel der bestmöglichen Sicherheit zu erreichen.« (Kommission Lagerung hoch radioaktiver Abfallstoffe 2016a: 31).

Aus diesen Zitaten wird klar, dass für die Kommission der Einbezug der Bevölkerung eine zentrale Stelle einnimmt, auch was die Revidierung von Entscheidungen angeht. Dies erfordert, dass wissenschaftliche Diskurse transparent geführt werden und die Öffentlichkeit daran mitwirkt, dass auch ihre Perspektive eingeholt wird. Neben der Notwendigkeit, die Möglichkeit zur Fehlerkorrektur vorzusehen, wird eine zukunftsethische Motivation angeführt. 
Der Verweis im Stand AG auf Reversibilität »nach Maßgabe der $\$ \$ 12 \mathrm{ff}$.« nimmt demgegenüber eine restriktive Haltung ein. Mit $\$ 12$ beginnt Teil 3 des Gesetzes, in dem die Verfahrensschritte der Standortauswahl beschrieben werden. Reversibilität wird dadurch an die Ablaufplanung gebunden, findet darin aber keine explizite Erwähnung. Aufgenommen sind in diesen Teil verschiedene Haltepunkte: Fachkonferenzen, Versammlungen, Bestimmungen durch Bundesgesetz. Nicht benannt ist, ob und, wenn ja, wie viele Verfahrensschritte zu welchem Zeitpunkt im Verfahren revidiert werden können. Ist es beispielsweise möglich, während der untertägigen Erkundung aufgrund neuer Erkenntnisse auf einen Verfahrensschritt vor dem Bundesgesetz zurückzuspringen, das die untertägig zu erkundenden Standorte bestimmt? Oder liegt der ungewöhnlichen Verfahrensplanung, die Zwischenentscheide per Bundesgesetz vorsieht, genau diese Motivation zu Grunde, Marken zu setzen, hinter die nicht zurückgegangen werden kann?

Es offenbart sich ein Spannungsfeld, das sich neben dem formulierten zukunftsethischen Anspruch der Kommission im Wesentlichen aus einem unterschiedlichen Verständnis von Sicherheit speist: Den Formulierungen der Endlagerkommission zu Reversibilität liegt offenkundig die Annahme zu Grunde, dass zum einen Beteiligung Sicherheit erhöht, zum anderen zur Gewährleistung auch technischer Sicherheit eine Fehlerkorrektur ohne Schranken möglich sein muss.

Dem steht die Verfahrensplanung des 2017 revidierten StandAG mit Beteiligung, Korrekturschleifen und anschließenden Bundesgesetzen diametral entgegen: Durch die Planung wird Beteiligung in bisher nicht gekanntem Umfang festgeschrieben, gleichzeitig aber auf genau diese partizipativen Elemente begrenzt. Mehr Beteiligung kann auf Grundlage des Gesetzes nicht eingefordert werden; weitergehenden Forderungen, sollten sie im Laufe des Verfahrens artikuliert werden, wird mit Bundesgesetzen ein nur schwer zu öffnender Riegel vorgeschoben. Darin kommt ein Sicherheitsverständnis zum Ausdruck, das technische Sicherheit auch durch Planungs- und Handlungssicherheit definiert: Der eingeschlagene Pfad kann auf diese Weise nicht vollständig in Frage gestellt werden, die Entsorgung läuft auf jeden Fall weiter und es wird die Gefahr eliminiert, wiederholt auf den Ausgangspunkt zurückgeworfen zu werden. Dadurch wird das Risiko in Kauf genommen, neue Erkenntnisse in ihrer Bedeutung für die Sicherheit der Entsorgung aufgrund von Pfadabhängigkeit zu negieren. Gleichzeitig wird das Risiko von Entsorgungsprokrastination, häufig durch die Formulierung wait and see beschrieben, verringert.

Kurz gefasst kann dieses Spannungsfeld auf die Frage reduziert werden, ob durch weitgehendes Offenhalten von Optionen wirklich der Sicherheit gedient wird, oder ob gerade durch Optionen der Reversibilität wie rückholbare Einlagerung Kompromisse eingegangen werden, die letztlich die Sicherheit reduzieren.

Durch die Reduktion des Reversibilitätsbegriffs auf die Mechanismen des gesetzlich fixierten Verfahrensablaufs wird auch der Wirkradius von Expert"innendissens eingeschränkt. Neue Erkenntnisse, die hinter getroffene Entscheidungen, insbesondere hinter solche, die durch Bundesgesetz bestimmt wurden, zurückweisen, können auf diese Weise nur schwer ins Verfahren eingespeist und umgesetzt werden; im schlimmsten Fall kann daraus eine Verringerung der Sicherheit, ausgelöst durch Pfadabhängigkeit, resultieren. 
Das Standortauswahlverfahren ist sicherlich resonanzfähig für Anpassungen; ob es hinsichtlich gesellschaftlicher Erwartungen an ein reversibles Verfahren einerseits und größerer Anpassung wie beispielsweise Kursänderungen oder Rückschritte andererseits flexibel genug aufgestellt ist, muss abgewartet werden.

\section{Expert*innendissens im Rahmen von Endlagerprojekten}

Die Endlagerung der hochradioaktiven Abfälle ist eines der technisch und finanziell aufwändigsten Infrastrukturprojekte in der Geschichte der Bundesrepublik Deutschland. Darüber hinaus ist sie ein Thema, das in der deutschen Gesellschaft kontinuierlich $\mathrm{zu}$ Kontroversen führt. Die Entsorgung der Altlast radioaktiver Abfälle hat eine weitere Altlast im Gepäck: Den seit ihrer Einführung schwelenden Konflikt über Nutzen und Gefahren der Energieerzeugung mittels Kernenergie.

Dieser Konflikt zeigt eine Reihe von Facetten: Die Gefahr des Missbrauchs auch ziviler Kerntechnik zum Bau von Nuklearwaffen, die Frage nach der Akzeptabilität einer Hochrisikotechnologie, toxische Abfallstoffe, deren langfristige Entsorgung nur durch dauerhafte Isolation vom Menschen und seiner Umwelt zu erreichen ist. Wissenschaftliche Expertise spielt in all diesen Facetten des Konfliktes eine Rolle. Wissenschaftliche Expert"innendissense haben nicht nur die Kernenergienutzung, sondern auch die Versuche zur Entsorgung der Abfälle stets begleitet.

Die nachfolgenden Fallstudien wurden ausgewählt, weil:

- der jeweilige wissenschaftliche Expert*innendissens öffentlich wahrnehmbar ausgetragen wurde, und somit grundsätzlich die Möglichkeit bestand, dass der Disput eine Wirkung auf den jeweiligen Prozess hatte,

- sie gut dokumentiert sind und damit die Möglichkeit bieten, die Dissense zu analysieren,

- sie jeweils unterschiedliche Verfahren und Standorte und unterschiedliche Sachverhalte adressieren.

Als einschränkend für die Übertragbarkeit muss angesehen werden, dass die untersuchten Expert"innendissense sich sämtlich auf spätere Verfahrensschritte der Endlagerung radioaktiver Abfälle beziehen und nicht auf den Standortauswahlprozess. Für die Beispiele aus der Bundesrepublik Deutschland ist das unter anderem darin begründet, dass in der Vergangenheit für kein Endlager ein dem aktuellen Standortauswahlverfahren vergleichbarer Prozess durchgeführt wurde. Das Beispiel aus Schweden wurde ausgewählt, weil schon die gute Dokumentation des Dissenses auf eine aus den deutschen Endlagerprojekten bisher nicht gekannte Kultur der Konfliktbewältigung hinweist und so einen Erkenntnisgewinn für das Standortauswahlverfahren in der Bundesrepublik verspricht. 


\section{Fallstudie Endlager Schacht Asse II}

Die Asse ist ein Höhenzug im Landkreis Wolfenbüttel, der durch Halokinese, Aufdomung von Salinargesteinen im tiefen Untergrund, gebildet wurde. Im ehemaligen Kalibergwerk Asse wurden von 1967 bis 1978 in 13 Kammern rund $47.000 \mathrm{~m}^{3}$ im Wesentlichen schwach- und mittelaktiver Abfälle der Bundesrepublik Deutschland eingelagert. Das Bergwerk wurde während dieser Zeit von der Gesellschaft für Strahlenforschung mbH München (GSF) als Forschungsbergwerk betrieben, in dem unter anderem verschiedene Einlagerungstechniken erprobt werden sollten. Tatsächlich »dient die Einlagerung ab 1971 der faktischen Endlagerung nahezu aller schwach- und mittelradioaktiven Abfälle der Bundesrepublik «. ${ }^{1}$ Zum gegenwärtigen Zeitpunkt sollen diese Abfälle aus dem Bergwerk zurückgeholt werden; im Sinne der Definition des Standortauswahlgesetzes (Deutscher Bundestag 2017) handelt es sich dabei um eine Bergung. Dazu wurde das Atomgesetz (Deutscher Bundestag 1959) durch das Gesetz zur Beschleunigung der Rückholung radioaktiver Abfälle und der Stilllegung der Schachtanlage Asse (Deutscher Bundestag 2013) ergänzt.

Es entwickelte sich schon früh ein Disput um die Eignung des Bergwerks Asse als Endlager; Klagen gegen den Standort und für die anschließende Entscheidung zur Rückholung folgten. So wurde beispielsweise die Sicherheitsstudie der GSF vom Bergamt Goslar »mit Vermerk vom 31.5.1967 massiv kritisiert (Bündnis 90/Die Grünen im niedersächsischen Landtag 2012). Ursächlich für den wissenschaftlichen Dissens wie auch leitend für den gesellschaftlichen Konflikt war die Frage eines möglichen Wasserzutritts in das Bergwerk mit Folgen wie Ablaugung des noch vorhandenen Salzes, Zutritt von Wasser zu den Einlagerungskammern, Mobilisierung eingelagerter Stoffe und deren Transport bis in die Biosphäre. In diesem Fall ist von einer radiotoxischen und chemotoxischen Gefährdung für Menschen und Umwelt auszugehen.

Durch den weitgehenden Salzabbau im Gewinnungsbetrieb, durch den die Kammern insbesondere an der Südflanke nur noch einen geringen Abstand zum Deckgebirge haben, und die geologische Situation der Asse konvergieren ${ }^{2}$ die Strecken und Kammern im Bereich der Südflanke des Höhenzugs sehr stark. Im Gutachten der CDM Smith Consult GmbH (CDM Smith Consult GmbH 2015: 7) zur Beurteilung der gebirgsmechanischen Gefährdungsanalyse des IfG der Abbaue 3 und 4 auf der 490-m-Sohle und des Abbaus 2 auf der 511-m-Sohle werden in diesem Zusammenhang exemplarisch die »für die Asse prägenden Wechselwirkungen zwischen den infolge von Entfestigung und Bruchprozessen nachgebenden Tragelementen und dem an Großstörungen beziehungsweise Scherbändern »nachrutschenden« südlichen Deckgebirge« betont.

Nach Metz (2016) wird »seit 1988 [...] ein anhaltender Lösungszutritt in etwa 500 bis $600 \mathrm{~m}$ Tiefe beobachtet. Die Zutrittsrate dieser an Steinsalz (Natriumchlorid) gesättigten Lösung ist mit rund zwölf Kubikmeter pro Tag seit 1992 in etwa konstant« (Metz

https://www.bge.de/asse/kurzinformationen/geschichte-der-schachtanlage-asse-ii

»Unter Konvergenz wird die ausbruchbedingte seitliche Profileinengung eines Hohlraums (Stauchung) aufgrund von Lastumlagerungen im umgebenden Cebirge verstanden. Die Profilaufweitung eines Hohlraums wird als Divergenz (Spreizung) bezeichnet.« (Prinz/Strauß: Ingenieurgeologie, 6. Auflage, S. 656 Kapitel 17 Tunnelbau, Springer Spektrum, Berlin) 
2016: 68). Die Bundesgesellschaft für Endlagerung mbH (BGE) stellt dar, dass durch Risse im Salz und im umliegenden Gebirge rund 13,5 $\mathrm{m}^{3}$ Grundwasser pro Tag in die Schachtanlage Asse II laufen und ein Absaufen der Asse, also ein Volllaufen des Grubengebäudes mit stetigem Anstieg des Wasserspiegels, nicht ausgeschlossen werden könne (Bundesgesellschaft für Endlagerung $\mathrm{mbH}$ 2019). Das Niedersächsische Landesamt für Bodenforschung (NLfB) weist in einem Gutachten 1964 noch Laugenzutritte von drei bis vier $\mathrm{m}^{3}$ pro Tag aus (NLfB 1964).

Der grundlegende tektonische Bau des Asse-Sattels war im Wesentlichen bekannt, als 1964/65 der Wechsel vom Gewinnungsbergbau zum Betrieb eines Forschungsbergwerks erfolgte. In mehreren Gutachten, unter anderem der Bundesanstalt für Bodenforschung (BfB 1963b, 1963a) und des NLfB (siehe oben), wurden keine schwerwiegenden Einwände gegen eine Eignung der Asse II als Endlager erhoben. Das »Gutachten über die Gefährdungsmöglichkeit der Trinkwasserversorgung der Gemeinden in der Umgebung des Kali-Bergwerkes Asse II durch Einlagerung von radioaktiven Abfällen in den aufgelassenen Grubenbauen« (Semmler 1965) weist zwar auf bestehende Wissenslücken und daraus resultierenden weiteren Forschungsbedarf hin, hatte aber keinen weiteren Einfluss auf die Inbetriebnahme des Forschungsbergwerks. Eine weitere wesentliche Grundlage für die Einlagerung im Auftrag des Bundesforschungsministeriums war die »Studie über die bisherigen Laugenzuflüsse auf den Asse-Schächten und die Gefahr eines Wasser- oder Laugeneinbruchs in das Grubengebäude des Schachtes II« der Gesellschaft für Strahlenforschung mbH München (GSF) und des Instituts für Tiefenlagerung Clausthal-Zellerfeld. Die Autoren kommen zu dem Schluss, »dass die Gefährdung für die Schachtanlage Asse II durch Wasser- oder Laugeneinbrüche als minimal anzusehen beziehungsweise mit an Sicherheit grenzender Wahrscheinlichkeit sogar auszuschließen« sei (Kühn et al. 1967: 32).

Zweifel an den positiven Einschätzungen zur Standfestigkeit des Grubengebäudes und den Gefahren eines Laugenzutritts wurden früh geäußert und auf die Notwendigkeit einer Verfüllung nicht genutzter Grubenbaue zur Stabilisierung hingewiesen. So hat beispielsweise das Oberbergamt Clausthal-Zellerfeld 1963 vom NLfB ein Gutachten erbeten, »in welchem Umfange über dem Grubengebäude des Schachtes Asse II und des Schachtes Asse I einschließlich beider Schächte aus geologischer und hydrologischer Sicht eine Gefährdung der Oberfläche eintreten kann und gegebenenfalls in welchem Umfange«. Das Landesamt führte in seinem Gutachten daraufhin die Laugenzutritte auf (siehe oben) und stellte Szenarien eines Absaufens des Grubengebäudes dar (Bündnis 90/Die Grünen im niedersächsischen Landtag 2012).

Erst mit der Arbeit »Atommülldeponie Salzbergwerk Asse II: Gefährdung der Biosphäre durch mangelnde Standsicherheit und das Ersaufen des Grubengebäudes« (Jürgens 1979), in der dargelegt wird, dass realistische Szenarien für die zukünftige Entwicklung des Grubengebäudes zu einem Transport radioaktiver Stoffe in genutzte Grundwasserleiter führen können, wird jedoch ein Dissens in der wissenschaftlichen Beurteilung der Asse offensichtlich.

Trotz eines öffentlich wahrnehmbaren wissenschaftlichen Disputs dauerte es bis zum Jahreswechsel 2008/2009 (BGE, 2019), bis die Schachtanlage Asse II unter Atomrecht gestellt und das Bundesamt für Strahlenschutz (BfS) zum Betreiber wurde. Ohne Experten wie Hans-Helge Jürgens, die gegen die Darstellung der Wissenschaftler der 
GSF und von ihr beauftragter Gutachter Stellung bezogen, hätte ein wirkungsvoller Widerstand gegen den Betrieb der Schachtanlage Asse II sehr viel schwerer aufrechterhalten werden können. Dem gegenüber steht die Tatsache, dass eine Änderung im Umgang mit dem früheren Forschungsbergwerk erst stattfand, als die naturwissenschaftlichen Fakten, im Wesentlichen die deutliche Zunahme der Laugenzutritte, die Prognosen der externen Expert*innen unterstützten. Gesellschaftlicher Widerstand hat letztlich zu einer Änderung im Umgang mit der Schachtanlage Asse II geführt; die Gegenexpertise hatte mittelbare beziehungsweise unterstützende Wirkung, um zu einer Revision und Neuausrichtung des Verfahrens zu gelangen.

\section{Fallstudie Gorleben}

Der wohl prominenteste wissenschaftliche Expert*innendissens im Zusammenhang mit dem Salzstock Gorleben, der von 1979 bis 2012 auf seine Eignung als Wirtsgesteinskörper für ein Endlager für hoch radioaktive Abfälle erkundet wurde, ist der Disput um die sogenannte Gorlebener Rinne. Der von der Physikalisch Technischen Bundesanstalt (PTB) mit der quartärgeologischen Untersuchung des Deckgebirges beauftragte Kieler Geologieprofessor Klaus Duphorn beschrieb in seinem Gutachten (Duphorn et al. 1983) eine subglazial gebildete, mit quartären Lockersedimenten gefüllte Rinne, die den Salzstock Gorleben quert und stellenweise Kontakt zum Salzspiegel hat.

Der Dissens betrifft die Bewertung der Gorlebener Rinne beziehungsweise deren Bedeutung für die Sicherheit eines möglichen Endlagers im Salzstock Gorleben. Protagonisten des Expert*innenstreits sind neben dem ursprünglichen Gutachter Duphorn im Wesentlichen Mitarbeiter*innen der Bundesanstalt für Geowissenschaften und Rohstoffe (BGR). Als exemplarisch für die Gegengutachten der BGR kann hier der Bericht von Keller (2009) angesehen werden. Während Duphorn in seinem Gutachten zu dem Schluss kommt, der Salzstock Gorleben habe »seine Eignungshöffigkeit als Endlager für hoch-, mittel- und schwachradioaktive Abfälle verloren« (Flachsbarth et al. 2013), stellt Keller zwar fest, dass »die Lage zukünftiger subglazialer Rinnenbildungen nicht vorhergesagt werden kann« und daher »die Deckgebirgsverhältnisse über dem Salzstock Gorleben als Zukunftsoption auch für alternative Standorte in Norddeutschland zu unterstellen« (Keller 2009) seien. »Wegen der in Salzstöcken akkumulierten großen Mächtigkeiten von Steinsalz« sei jedoch »eine Abfalleinlagerung in Tiefenbereichen von 800 - $1000 \mathrm{~m}$ vorgesehen, die zukünftige negative Einwirkungen auf ein Endlager durch Rinnenbildungen« (Keller 2009, S. 19) ausschließe.

Der sich an das Gutachten Duphorns anschließende Konflikt geht über den wissenschaftsinternen Dissens hinaus. So wurde am 3. August 1982 vom Deutschen Atomforum ein interner Vermerk des Bundesministeriums für Forschung und Technologie (BMFT) veröffentlicht, in dem das Fazit der Arbeit Duphorns »als unwissenschaftlich und weit über dessen Fachgebiet hinausgehend« bezeichnet wird (Flachsbarth et al. 2013). Im Rahmen des parlamentarischen Untersuchungsausschusses (2009 bis 2012) bestätigte der Zeuge Dr. Wolf von Osten, ab April 1982 Referatsleiter im BMFT, »dass Prof. Dr. Klaus Duphorn beim BMFT in Ungnade gefallen war, weil er zu Erkenntnis- 
sen wissenschaftlicher Art gekommen war, die sich nicht mit der politischen Linie des Hauses deckten« (Flachsbarth et al. 2013).

Ungeachtet des wissenschaftlichen Dissenses wurde im Salzstock Gorleben ein Forschungsbergwerk aufgefahren und der Salzstock von 1979 bis 2000 und von 2010 bis 2012 auf seine Eignung als Wirtsgestein für ein Endlager für hoch radioaktive Abfälle untersucht ${ }^{3}$. Niederschlag gefunden hat der Dissens letztlich im Abschlussbericht der Kommission Lagerung hoch radioaktiver Abfallstoffe, die im Zusammenhang mit Abwägungskriterien für die Standortauswahl feststellt, dass ein Deckgebirge, das den einschlusswirksamen Gebirgsbereich zusätzlich vor glazialen Rinnen schützt, die Robustheit des Endlagersystems steigert (Kommission Lagerung hoch radioaktiver Abfallstoffe 2016a: 54). Auch die Mindestanforderungen im Standortauswahlgesetz (Deutscher Bundestag 2017) greifen die Schutzfunktion des Deckgebirges gegenüber eiszeitlich bedingter Erosion auf. Im September 2020 wurde der Salzstock Gorleben-Rambow im Zwischenbericht Teilgebiete, dem ersten Schritt im Standortauswahlverfahren nach dem StandAG, nicht als Teilgebiet benannt und scheidet damit gemäß $\$ 36$ Abs. 1 Nummer 1 StandAG aus dem Verfahren aus. Der Ausschluss wird im Wesentlichen durch die ungünstige Bewertung des Abwägungskriteriums »Deckgebirge« begründet. Dieses Kriterium wurde zudem durch die Bearbeiter "aufgrund der geringen Tiefe des Strukturtops [...] stärker gewichtet« (Bundesgesellschaft für Endlagerung mbH 2020).

\section{Fallstudie Schweden: Kupferkorrosion unter anaeroben Bedingungen}

Das schwedische Entsorgungskonzept für ausgediente Brennelemente sieht die Einlagerung in einem Gusseisenbehälter vor, der zum Schutz vor Korrosion mit einer $5 \mathrm{~cm}$ dicken Schicht aus Kupfer ummantelt ist (Strömberg et al. 2019). Die Behälter sollen in einem kristallinen Wirtsgestein in etwa 500 Meter Tiefe eingelagert werden, eingebettet in eine Lage von Bentonit, einem Lockergestein, das im Wesentlichen aus quellfähigen Tonmineralen besteht und den Zutritt von Wasser zu den Behältern erschweren soll. Als Standort für das Endlager wurde Forsmark ausgewählt, ein kleiner Ort etwa $115 \mathrm{~km}$ nördlich von Stockholm.

Von Wissenschaftlern der Schwedischen Königlich Technischen Hochschule (KTH) wurde zuerst 1986 (Hultquist 1986) und ein weiteres Mal 2009 (Hultquist et al. 2009) in Experimenten gezeigt, dass Kupfer in sauerstofffreiem Wasser unter Bildung von Wasserstoff reagiert. Diese Beobachtung wurde als Anzeichen für Korrosion interpretiert. Die schwedische Betreibergesellschaft SKB führte daraufhin Expert*innenworkshops und Experimente durch, um das Problem nachzuvollziehen. Man stellte fest, dass die Bildung von Wasserstoff in diesem Prozess nicht mit den Korrosionsprodukten des Kupfers korreliert (Hedin et al. 2018).

Der Expert*innendissens wird in Schweden sehr transparent geführt. Veröffentlichungen werden mit Reviews der jeweiligen Gegenexpert*innen beantwortet. Auch der

Zwischen 2000 und 2010 wurden die Erkundungsarbeiten im Rahmen eines Moratoriums eingestellt, seit 2012 befindet sich das Bergwerk im Offenhaltungsbetrieb. 
schwedische Kärnavfallsrådet, der die Regierung in Fragen der Entsorgung radioaktiver Abfälle berät, setzt sich in mehreren Publikationen mit dem Thema auseinander (Kärnavfallsrådet 2009, 2010).

Beide Seiten teilen die Beobachtung der Wasserstoffbildung, kommen jedoch $\mathrm{zu}$ jeweils unterschiedlichen Analysen hinsichtlich der Ursache. Entsprechend unterscheiden sich auch die Bewertungen bezüglich der Bedeutung des beobachteten Phänomens für die Sicherheit des schwedischen Endlagerkonzepts. Neben den Hauptakteuren, der KTH Forschungsgruppe auf der einen Seite und den Wissenschaftlern von SKB auf der anderen Seite, haben sich eine Reihe weiterer Institutionen, im Wesentlichen Forschungseinrichtungen, mit der Thematik auseinandergesetzt.

Der öffentlich ausgetragene Dissens hat dazu geführt, dass das Genehmigungsverfahren zur Errichtung eines Endlagers für mehrere Jahre ins Stocken geraten ist. Aktuell steht nach den Stellungnahmen von SKB, der Aufsichtsbehörde und des Umweltgerichts die Entscheidung der Regierung über die Genehmigung aus. Der Dissens hat weitere Forschung angeregt und Einfluss auf politische Entscheidungen entfaltet.

Der Expert*innendissens wird weitgehend produktiv und sachlich geführt. Während in den oben beschriebenen Beispielen aus der deutschen Entsorgungsgeschichte der Expert*innendissens Teil einer größeren, generellen Debatte um eine Entsorgungsoption oder ein Entsorgungsverfahren ist, kann die aktuelle Diskussion in Schweden um mögliche Korrosion von Kupfer unter den Umgebungsbedingungen eines Endlagers vordergründig als rein wissenschaftliche Debatte wahrgenommen werden. Obwohl er Auswirkungen auf das Genehmigungsverfahren hat, wird der Expert"innenstreit, soweit beobachtbar, nicht zur Unterstützung einer bestimmten politischen Agenda instrumentalisiert. Die Kommunikation über den Sachverhalt ist im Gegenteil durch Offenheit und grundsätzliche Anerkennung der Expertise der jeweiligen Antagonisten geprägt.

\section{Reflexion der drei Fallstudien}

Die hier skizzierten Fallstudien aus dem Bereich der Endlagerung radioaktiver Abfälle unterscheiden sich nicht nur hinsichtlich der Gegenstände, um die sich der jeweilige Dissens dreht, sondern auch in ihrer Wirkung auf das entsprechende Endlager oder Verfahren, der Wahrnehmbarkeit in der Öffentlichkeit, der Weise, wie der Dissens ausgetragen wird und der Rollen, die die beteiligten Expert*innen einnehmen. Die Dispute um die Gorlebener Rinne und die Stabilität des Bergwerks Asse II stellen jeweils die Eignung eines Standortes als Endlager in Frage. Der Disput um die Korrosion von Kupfer reicht insofern weiter, als er eine wesentliche technische Komponente und damit implizit das gesamte nationale Endlagerkonzept Schwedens auf den Prüfstand stellt.

In allen Fallstudien ist der dargestellte Expert*innendissens nur ein Teil eines umfassenderen Konfliktfeldes. Im Fall der Asse stand und steht der Vorwurf im Raum, die Wahl des Bergwerks als Endlager sei nicht aufgrund von Eignungskriterien und zur Verwendung als Forschungsbergwerk erfolgt, sondern der Tatsache geschuldet, dass für die sich entwickelnde Atomindustrie und Kernforschung in Deutschland eine Entsorgungslösung geschaffen werden musste und die Asse »als Teil des Entsorgungsvorsor- 
genachweises « benötigt wurde (Bündnis 90/Die Grünen im niedersächsischen Landtag 2012). Der Untersuchungsausschuss des Niedersächsischen Landtages kommt zu diesem Schluss, und verschiedene Quellen stützen diese Annahme; exemplarisch soll hier die folgende Passage aus der Notiz der Projektgruppe Endlagerung radioaktiver Abfälle der Karlsruher Gesellschaft für Kernforschung mbH zur Besichtigung der Schachtanlage Asse der Wintershall AG angeführt werden: „Die Abbaue sind von oben her zugänglich, beziehungsweise ein Zugang ist leicht zu schaffen. Daher können die Abfälle behälterlos verstürzt werden, so dass das Einbringen einfach und wenig kostspielig wird « (Projektgruppe Endlagerung r.a. Abfälle 1964). Der Schluss liegt nahe, dass es hier weniger um kostengünstige Forschung als um die Entsorgung radioaktiver Abfälle geht. Eine behälterlose Einlagerung erschwert eine spätere Untersuchung im Rahmen von Forschung erheblich, weil die Abfälle nur noch unter hohem Risiko für das Personal, insbesondere hinsichtlich Strahlenschutz, gehandhabt werden können. Eine Entfernung aus dem Bergwerk nach Beendigung der Forschungsarbeiten ist so ebenfalls nur schwer umsetzbar.

Die Fallstudie zur Asse zeigt auch, dass interessengeleitete Dissense nicht allein qua Dissens zu Halt und Rückschritt (also zu einer Realisierung der Reversibilitätsoption) führen müssen. Vielmehr muss gesellschaftlicher Druck unterstütztend wirken. (Kasperson et al. 1988) sprechen im Rahmen von Risiken und ihrer Wahrnehmung von ripple effects. Das heisst, ein Risiko/ein Ereignis entfaltet nicht per se gesellschaftliche Relevanz (beziehungsweise Resonanz). Dazu müssen insbesondere die Medien und Diskurse in verschiedenen Arenen (siehe dazu auch den Beitrag von Losada et al. »Arenen zur Austragung von Dissensen in der Endlagerpolitik« in diesem Band) Informationen in der Gesellschaft weitertragen. Gegenexpertise und gesellschaftliche Kräfte können erst gemeinsam dazu führen, dass Reversibilität in Anspruch genommen wird. Durch Dissense können, wie oben erwähnt, Fakten und Aspekte bewusst gemacht und in Diskurse integriert werden. Diese müssen dann aber auch gesellschaftlich weitere Wellen schlagen, wie ein Stein, der in einen See fällt, wobei sich die Wellen weiter ausbreiten.

In Gorleben wurde der Konflikt durch den ursprünglichen Plan initiiert, dort ein nukleares Entsorgungszentrum der Bundesrepublik zu errichten, das neben einem Endlager auch weitere kerntechnische Anlagen zum Unterhalt des sogenannten Brennstoffkreislaufs beherbergen sollte. Der Standortauswahl 1977 war eine geowissenschaftliche Untersuchung zu möglichen geeigneten Standorten in Niedersachsen vorausgegangen, bei der Gorleben als Standort bereits ausgeschieden war. Nachdem der damalige niedersächsische Ministerpräsident den Salzstock in Gorleben als einzig geeigneten in Niedersachsen benannt hatte, wurde vom Präsidenten der Bundesanstalt für Geowissenschaften und Rohstoffe klargestellt, dass diese Aussage nicht auf Wissenschaftler*innen der BGR zurückzuführen sei (Kommission Lagerung hoch radioaktiver Abfallstoffe 2016b).

Auch der Konflikt um die Kupferkorrosion in Schweden verweist auf einen weiterreichenden Zusammenhang. Die Möglichkeit einer Korrosion der für die Endlagerung vorgesehenen Behälter stellt das gesamte KBS-3-Konzept in Frage, auf dem die Entsorgungsstrategien in Schweden und auch in Finnland beruhen. Da geeignete Sedimentgesteine, wie sie in anderen Staaten, beispielsweise in Frankreich, Belgien oder der Schweiz, als Wirtsgestein für ein Endlager genutzt werden sollen, in Skandinavien nicht 
zur Verfügung stehen, stünde damit auch die Idee der untertägigen Endlagerung insgesamt zur Disposition. ${ }^{4}$ Die schwedische NGO Miljöorganisationernas kärnavfallsgranskning (MKG), die das KBS-3-Konzept kritisch hinterfragt, unterstützt darüber hinaus schon seit längerer Zeit die Option einer Endlagerung in tiefen Bohrlöchern statt in einem Bergwerk. Am Expert*innendissens nimmt sie nur insofern teil, als sie über die jeweiligen Ergebnisse berichtet und in engem Kontakt mit der Strahlenschutzbehörde SSM den Prozess begleitet; eigene Expertise bringt sie jedoch nicht in den Diskurs ein.

Alle Akteur"innen in den dargestellten Expert"innendissensen sind Wissenschaftler*innen. Das gilt auch für andere Dispute im Zusammenhang mit den dargestellten Endlagerprojekten sowie weitere betrachtete Projekte. Wie oben (Abschnitt 1) erwähnt, ist Laienexpertise in der Endlagerung nach diesen Beobachtungen nicht oder nur untergeordnet von Bedeutung. Dennoch spielen weitere Akteur*innen, wenn nicht direkt in den Expert"innendissensen, so doch in deren Umfeld eine Rolle. Das können die Auftraggeber*innen von Expert*innen sein, z.B. Betreiber, Aufsichtsbehörden, Ministerien, Bürgerinitiativen, aber auch Beschwerdeführer in Erörterungs- oder Klageverfahren. In direkter oder indirekter Weise können diese Stakeholder Einfluss auf den Expert*innenstreit und damit auf das Verfahren ausüben. Zudem ist damit zu rechnen, dass etwa in Regionalkonferenzen beteiligte Bürger*innen zu Bürgerexpert*innen werden, die sich zu ihrem privaten Wissen auch Grundkenntnisse der Endlagerung aneignen.

Expert* innen müssen nicht immer im Auftrag handeln. So veröffentlichten die Wissenschaftler*innen der KTH in Schweden ihre Untersuchungen ohne Auftrag. Im Fall der Gorlebener Rinne war der Experte von Behördenseite beauftragt; um seine Einschätzung und das zugrundeliegende Gutachten in Frage zu stellen, wurden weitere Gutachter*innen von Behörden beauftragt. Im oben geschilderten Expert*innendissens um die Schachtanlage Asse II handelte ein Gutachter im Auftrag der Betreibergesellschaft, ein anderer wurde von einer Bürgerinitiative beauftragt.

Die Zuschreibung der Expert*inneneigenschaft folgt in den ersten Fällen praktisch aus der Beauftragung: Als Gutachter*in wird beauftragt, wer vom Auftraggeber als Expert"in anerkannt wird. Die Wissenschaftler*innen der KTH, die ihre Expertise ungefragt in die Debatte einbrachten, werden aufgrund ihrer Stellung als Lehrende oder wissenschaftliche Mitarbeiter*innen der Hochschule als Expert*innen anerkannt sowie durch die Tatsache, dass ihre Arbeit in der Öffentlichkeit Gehör findet. Darüber hinaus werden die durchgeführten Experimente und dargestellten Beobachtungen insofern anerkannt und gewürdigt, als sie von anderen Beteiligten wiederholt und überprüft werden und daraus weiterer Forschungsbedarf abgeleitet wird. Schlussendlich muss auch die Unterbrechung des Verfahrens als Anerkennung der Expertise gewertet werden. te zur Aufnahme eines Endlagers für hochradioaktive Abfälle angesehen. Steinsalz und Tongestein entstehen sedimentär durch chemische Fällung (Steinsalz) beziehungsweise physikalische Ablagerung (Tongesteine) in aquatischen, marinen oder terrestrischen, Millieus. Kristallingesteine werden durch Kristallisation aus einer magmatischen Schmelze oder durch Umkristallisation präexistierender Gesteine im Rahmen einer Metamorphose gebildet. 


\section{Typen von Expert*innendissens}

Auf Grundlage von Praxisbeispielen ${ }^{5}$, von denen drei kurz vorgestellt wurden (siehe oben), und unter Zuhilfenahme von Literaturstudien wurden drei Typen von Expert* innendissens identifiziert: Erkenntnis-Dissens, fachlicher Dissens und interessengeleiteter Dissens.

Erkenntnis-Dissens geht häufig großen Veränderungen des Wissensstandes voraus. Neue grundlegende Erkenntnisse verändern nicht nur die menschliche Wahrnehmung der Welt, sondern auch den Umgang mit ihr. Sie haben dementsprechend großen Einfluss, können zu sozialen und politischen Veränderungen führen und stoßen in der Regel auf starken Widerstand. Häufig setzen sie sich erst dann durch, wenn die Beweise für die formulierte These auch für Laien offensichtlich zu Tage treten und die neue Interpretation der zu Grunde liegenden Beobachtungen nicht mehr in Frage gestellt werden kann. Es geht also um die Frage, wann neues Wissen tatsächlich breite Anerkennung findet (Kuhn 1970). Erkenntnis-Dissens kann daher auch als eine spezielle Form des fachlichen Dissenses aufgefasst werden. Als Beispiel für einen bedeutenden Erkenntnis-Dissens in den Geowissenschaften kann die Theorie der Plattentektonik angeführt werden. Von Alfred Wegener 1915 postuliert, dauerte es bis in die 1970er Jahre, bis die Erkenntnis, dass die Erdoberfläche nicht statisch ist, sondern aus mobilen Krustenplatten besteht, allgemein anerkannt wurde. Die Theorie beruhte auf nachvollziehbaren Beobachtungen; Wegener konnte jedoch keine plausible Ursache für eine Drift von Kontinentalplatten benennen. Erst die Weiterentwicklung der geowissenschaftlichen Methodik und die Erforschung der Ozeanböden nach dem Ende des zweiten Weltkriegs lieferten weitere Hinweise auf die Kontinentaldrift und schließlich die Beobachtung des sea floor spreading, der Neubildung von Ozeanboden an den mittelozeanischen Rücken und der Subduktion der ozeanischen Kruste an den Rändern der Kontinentalplatten.

Interessengeleiteter Dissens ist dadurch gekennzeichnet, dass wissenschaftliche Erkenntnisse artikulierten politischen, wissenschaftlichen oder anderen Interessen entgegenstehen. Daher werden sie nach ihrer Publikation durch Gegengutachten gezielt in Frage gestellt.

Es kann jedoch selbstverständlich nicht bei jedem Aufeinandertreffen von Expertise und differierender Expertise von einem interessengeleiteten Dissens ausgegangen werden. Ein weiteres, häufig zu beobachtendes, Merkmal eines interessengeleiteten Dissenses ist die gleichzeitige wissenschaftliche Unterstützung der zuvor artikulierten Interessen der Auftraggeber*innen durch das Gegengutachten. Darüber hinaus wird die Veröffentlichung von Gegenexpertise häufig von dem Versuch begleitet, Expert*innen, die die ursprüngliche wissenschaftliche Aussage veröffentlicht oder unterstützt haben, als unglaubwürdig darzustellen und ihre Expertise und Qualifikation

5 Als weitere Beispiele von Expert*innendissensen aus dem Bereich der Endlagerung radioaktiver Abfälle können die Frage der Bildung von kryogenen Klüften, der Eignung einer Überfahrungssohle für das Monitoring eingelagerter Abfälle, verschiedene Detailfragen im Zusammenhang mit dem Erkundungsbergwerk in Gorleben wie die sicherheitstechnische Bewertung der Durchfahrung von Anhydrit und die Bedeutung von Kohlenwasserstoffvorkommen genannt werden. 
in Zweifel zu ziehen. Dieser Typ kann anhand des Expert*innendissenses hinsichtlich der Eignung der Schachtanlage Asse II als Endlager für radioaktive Abfälle charakterisiert werden. Die Ergebnisse des parlamentarischen Untersuchungsausschusses legen nahe, dass der Einlagerung radioaktiver Abfälle im Bergwerk Asse wirtschaftliche Interessen zugrunde lagen. Die Expertise der Gegenexpert"innen wurde in Frage gestellt, auf die Veröffentlichung seiner/ihrer Studie mit Gegendarstellungen in der Lokalpresse reagiert.

Auch der oben beschriebene Disput über die Gorlebener Rinne trägt Züge eines interessengeleiteten Konflikts, beispielsweise hinsichtlich des Umgangs mit der unliebsamen Expertise der Kieler Wissenschaftler*innen und mit Blick auf die Tatsache, dass die politische Standortentscheidung sogar von der fachlich zuständigen Bundesanstalt direkt in Frage gestellt wurde. Gleichzeitig tragen der Dissens über die Gorlebener Rinne wie auch weitere in der Folge im Zusammenhang mit dem Erkundungsbergwerk in Gorleben aufgetretene Expert*innenstreite auch Merkmale eines fachlichen Dissenses.

Als fachlicher Dissens soll der wissenschaftliche Disput an sich bezeichnet werden. Dabei geht es um die unterschiedliche Analyse oder Bewertung von Beobachtungen, Sachverhalten etc. durch Wissenschaftler*innen einer oder mehrerer Disziplinen. Der fachliche Dissens kann entsprechend der untenstehenden Tabelle weiter untergliedert werden.

Ausgangspunkt ist jeweils eine spezifische Beobachtung, über die Einigkeit besteht. Im ersten Fall (F 1 ) sind sich die Expert*innen A und B sowohl über die jeweilige Beobachtung - Beobachtung A - als auch deren Analyse - Analyse A - einig. Dissens besteht hinsichtlich der Bewertung: Expert*in A kommt zu Bewertung A, Expert*in B zu Bewertung B.

Im zweiten dargestellten Fall (F 2) besteht ebenfalls kein Dissens hinsichtlich der Beobachtung, jedoch wird diese von den beiden Expert*innen unterschiedlich interpretiert. Davon abhängig kommen beide Expert*innen zu divergierenden Bewertungen. Selbst wenn beide zu dem Schluss gelangen, dass für die betrachtete Fragestellung die Beobachtung irrelevant sei, werden sich die Bewertungen dennoch aufgrund der unterschiedlichen Analysen unterscheiden.

Tabelle 1: Zwei Varianten des Typs fachlicher Expert *innendissens in Relation zu Beobachtung, Analyse und Bewertung

\begin{tabular}{|l|l|l|l|l|}
\hline & & Beobachtung & Analyse & Bewertung \\
\hline & Expert*in A & Beobachtung A & Analyse A & Bewertung A \\
\cline { 2 - 5 } & Expert*in B & Beobachtung A & Analyse A & Bewertung B \\
\hline & Expert*in A & Beobachtung A & Analyse A & Bewertung A \\
\cline { 2 - 5 } & Expert*in B & Beobachtung A & Analyse B & Bewertung B \\
\hline
\end{tabular}

Quelle: Eigene Darstellung

Fachlicher Dissens und die Unterscheidung in die dargestellten zwei Fälle lässt sich anhand der Dispute um die Gorlebener Rinne und die Frage der Kupferkorrosion illus- 
trieren. Die Gorlebener Rinne ist eine unter allen Expert*innen konsentierte Beobachtung. Auch die Analyse, der zufolge es sich um eine subglazial gebildete Rinne handelt, die mit Lockersedimenten gefüllt ist und Grundwasser führt, wird geteilt. Der Dissens dreht sich im Wesentlichen um die Bewertung von Beobachtung und Analyse: Um die Frage, ob die Grundwasserführung innerhalb des Nachweiszeitraums für ein Endlager zu einer sicherheitsrelevanten Schädigung des Wirtsgesteins führen kann; um die Frage, ob zukünftige klimatische Entwicklungen zu einer stärkeren Vereisung führen können, als aus den Zeugnissen der quartären Eiszeiten in Norddeutschland rekonstruiert; um die Frage, ob infolgedessen eine Bildung subglazialer Rinnen möglich ist, die die Tiefe eines Endlagers im Salzstock Gorleben erreichen. Demnach kann von einem fachlichen Dissens entsprechend Fall 1 der oben dargestellten Typologie gesprochen werden.

Im Expert"innendissens um die Korrosion von Kupfer unter anoxischen/anaeroben Bedingungen wird lediglich die Beobachtung von den Expert*innen geteilt. Hinsichtlich der Analyse herrscht, wie oben dargestellt, Dissens. Das ist in diesem Fall besonders bemerkenswert, weil ein Beweis für eine der möglichen Erklärungen der beobachteten Wasserstoffbildung bisher noch aussteht. Dissens besteht dennoch nicht nur hinsichtlich der Analyse, sondern auch der Bewertung des Phänomens mit Bezug auf die Sicherheit des schwedischen Endlagerkonzepts. Einen solchen Konflikt bezeichnen wir als fachlichen Expert"innendissens F2.

Die vorgestellte Typologie hilft, zu verstehen, dass es unterschiedliche Dissensarten gibt. Es kommt durchaus auf den Kontext an, etwa ob ein Streit lediglich wissenschaftsintern verläuft, oder ob sich außerwissenschaftliche Akteure daran beteiligen, ob er real und relevant ist oder vor allem instrumentelle Funktion hat. Sonst verwundert vielleicht, dass in einem Fall ein Dissens hohe Wellen schlägt und die Öffentlichkeit markant verunsichert, in einem anderen, quasi subkutan, Fakten unterschiedlich bewertet werden, ohne dass die Öffentlichkeit davon Notiz nimmt. Beispielsweise scheint, nach unserer Analyse der Fallstudien, von Interesse geleiteter Dissens nicht dauerhaft erfolgreich zu sein. Gerade Intransparenz bezüglich dessen, was dem öffentlich geführten Dissens wirklich zugrunde liegt (fachlicher Disput, der typisch für den Forschungsalltag ist, oder etwa gezielte Stimmungsmache und Überbetonung der Unsicherheiten) kann längerfristig das Verfahren vergiften. Insbesondere, wenn über die wirklichen Ziele spekuliert werden kann (im Sinne politischer Einflussnahme). Das Ziel ist also, mit der Typologie besser zu verstehen, wann Resonanz erzeugt und damit Wirkung ausgeübt wird. Dazu stellt sich die Frage, ob eine kritische Masse an Akteur*innen nötig ist, die sich mit dem Dissens beschäftigen und bestimmte Interessen verfolgen. Je nach dem, kann sich die Wirkung auf die öffentliche Wahrnehmung unterschiedlich ausprägen. Fachlicher Dissens kann, wie am schwedischen Beispiel gezeigt, Anlass zu Handlungen in einem reversiblen Verfahren werden. Interessengeleitete Dissense haben, wie an den Beispielen Asse und Gorleben gezeigt, das Potential, Risiken und Probleme über lange Zeit zu kaschieren und damit notwendiges Handeln zu verzögern oder zu unterbinden. 


\section{Schlussfolgerungen}

Die Beschäftigung mit Expert*innendissensen aus dem Umfeld der Endlagerung hat zunächst gezeigt, dass es verschiedene Typen von Dissensen gibt und offensichtliche Unterschiede dahingehend auftreten, auf welche Weise sich ein Dissens auf ein Verfahren auswirkt. Dabei reicht die Bandbreite der Wirkungen von der bloßen Unterstützung einer Position in einem gesellschaftlichen Konflikt (Fallbeispiel Asse) über die Berücksichtigung in Regelwerken (Fallbeispiel Gorleben) bis zum Halt in einem Verfahren und der Anregung von Forschungsaktivität zur Klärung des Disputs (Fallbeispiel Kupferkorrosion). Während in Schweden der fachliche Dissens relativ nüchtern verhandelt wird, stellt sich dies beim interessengeleiteten Dissens im Fall der Asse anders dar. Entlang der oben entwickelten Typologie kann angenommen werden, dass Erkenntnisdissens grundsätzlich erst ab einer gewissen Menge von Protagonisten und medialer Aufmerksamkeit der jeweils neuen Deutungsrichtung in der Öffentlichkeit wahrgenommen wird.

Fachlicher Expert*innendissens findet dabei in unserer Fallstudie und wahrscheinlich insgesamt relativ häufig unterhalb dieser Wahrnehmungsschwelle statt. Dies gilt umso mehr, wenn der Dissens sehr detaillierte Fragestellungen betrifft, die sich nur mit einem hohen Grad an fachlicher Vorbildung nachvollziehen lassen. Dies gilt auch im Fall, dass er wissenschaftsintern ausgetragen und nicht, wie im Beispiel der Kupferkorrosion, bewusst öffentlich kommuniziert wird. Fachlicher Dissens kann im Verfahren dann Wirkung zeigen, wenn er entweder in der Öffentlichkeit wahrgenommen wird, so dass man ihn nicht übergehen kann, oder das Verfahren beziehungsweise das Infrastrukturprojekt eine bestimmte Größe hat, so dass die Entscheider*innen unbedingt auf die Expertise von Fachabteilungen angewiesen sind. Auf diese Weise kann fachlicher Dissens zu Verzögerungen oder Halten im Verfahren führen, bis eine Klärung herbeigeführt wird, und letztlich, falls erforderlich, auch zu einem Umsteuern.

Der interessengeleitete Dissens schließlich wird seiner Natur nach von mindestens einer/m der beteiligten Expert*innen mit dem Ziel der Beeinflussung von Verfahren und Policies geführt. Das bedeutet auch, dass unter Umständen die öffentliche Wahrnehmbarkeit des Prozesses sogar notwendig ist, um das angestrebte Ziel zu erreichen.

Im Zusammenhang mit einem bewusst reversibel angelegten Verfahren bliebe $\mathrm{zu}$ untersuchen, ob verschiedene Dissenstypen unterschiedliche Auswirkungen darauf haben können, und woran dies liegt. In der Vergangenheit haben interessengeleitete Dissense, die nicht aufgelöst wurden, auch außerhalb Deutschlands hohe soziale und finanzielle Folgeschäden verursacht ${ }^{6}$. In das Standortauswahlgesetz sind nicht zuletzt Lehren aus dieser Vergangenheit eingegangen. Partizipation im Vorfeld von Entscheidungen und Transparenz können dazu beitragen, Konflikte zu vermeiden oder zu mindern, und das Vertrauen in demokratische Verfahren stärken. Expert*innendissens kann durch Einbindung in den Dialog fruchtbar gemacht werden. Darüber 
hinaus kann durch einen offenen und proaktiven Umgang mit Expert*innendissens auch dem Anspruch, wissenschaftsbasiert und transparent vorzugehen, Rechnung getragen werden.

Gleichwohl ist zu erwarten, dass die Wirkungen von Expert*innendissensen bis zu einem gewissen Grad unvorhersehbar bleiben. Umso wichtiger erscheint es, auf das Unvorhersehbare vorbereitet $\mathrm{zu}$ sein und das Verfahren entsprechend zu gestalten. Konzepte wie Resilienz (Lewinski 2016; Hoffmann 2017) oder Antifragilität (Taleb 2013) beschreiben dies. Dabei ist zu fragen, wo die Grenze von nötiger Offenheit zu Beliebigkeit gerade in einem von langfristiger Planung gezeichneten Verfahren ist.

Niederberger und Wassermann beschreiben in ihrem Buch Methoden der Stakeholdereinbindung und streifen auch das Thema Expert*innendilemma. Sie konstatieren, dass »viele Methoden der Expert*innen- und Stakeholdereinbindung [...] auf den Dialog ausgelegt [sind]. Damit wird die Hoffnung verbunden, dass durch den direkten Austausch positive Gruppenprozesse initiiert werden und so ein umfassenderes Bild über den Gegenstandsbereich möglich ist. Dabei muss aber klar sein, dass Expert*innen unterschiedliche Standpunkte und Sichtweisen vertreten, die mitunter ideologisch überformt sind [...]. In solchen Fällen kann eine konstruktive und ergebnisorientierte Diskussion zwischen den Expert*innen schwierig sein. Eine kompetente, qualifizierte und neutrale Moderation kann diese Gefahr abmildern, ist aber kein Garant für einen erfolgreichen Dialog« (Niederberger und Wassermann 2015, S. 44). Gerade auch im Hinblick auf die oben genannte Wirkung von Dissens auf die Öffentlichkeit erscheint dieser Hinweis auf professionelle Moderation des Verfahrens relevant. Eine entsprechend aktive Einbettung von Expert*innendiskursen in die öffentliche Partizipation erscheint daher geraten. Wir sehen dies als enorme Herausforderung angesichts der Geschichte der Kernenergie und der gescheiterten Endlagerprozesse in Deutschland. Der gesetzliche Rahmen durch das StandAG ist eine Voraussetzung, aber noch keine Garantie für einen gelingenden Prozess.

\section{Literatur}

Aichholzer, Georg/Bora, Alfons/Bröchler, Stephan/Decker, Michael/Latzer, Michael (Hg.) (2010): Technology Governance: Der Beitrag der Technikfolgenabschätzung. 1. Auflage. Baden-Baden: Nomos Verlagsgesellschaft mbH \& Co. KG.

Aparicio, Luis (2010): Making nuclear waste governable. Deep underground disposal and the challenge of reversibility. Springer.

BfB (1963a): Bericht zur Frage der Möglichkeiten der Endlagerung radioaktiver Abfälle im Untergrund. Bundesanstalt für Bodenforschung. Hannover.

BfB (1963b): Geologisches Gutachten über die Verwendbarkeit der Grubenräume des Steinsalzbergwerkes Asse II für die Endlagerung radioaktiver Abfälle. Hannover.

Bogner, Alexander (2010): Die Kultivierung des Dissenses. Ethik als GovernanceSemantik in Technikkontroversen. In: Aichholzer, Georg/Bora, Alfons/Bröchler, Stephan/Decker, Michael/Latzer, Michael (Hg.): Technology Governance: Der Beitrag der Technikfolgenabschätzung. 1. Auflage. Baden-Baden: Nomos Verlagsgesellschaft mbH \& Co. KG, 175-186. 
Bogner, Alexander/Menz, Wolfgang (2010): Konfliktlösung durch Dissens? Bioethikkommissionen als Instrument der Bearbeitung von Wertkonflikten. In: Feindt, Peter H./Saretzki, Thomas (Hg.): Umwelt- und Technikkonflikte. Wiesbaden: VS Verlag für Sozialwissenschaften, 335-353.

Böschen, Stefan (2000): Transdisziplinäre Forschungsprozesse und das Problem des Nicht-Wissens - Herausforderungen an Wissenschaft und Politik. In: Nachhaltige Entwicklung und Transdisziplinarität. In: Analytica; (16): 47-66.

Bundesgesellschaft für Endlagerung mbH (2019): Warum läuft Wasser in die Asse? htt ps:/www.bge.de/de/asse/themenschwerpunkte/themenschwerpunkt-das-wasserin-der-asse/warum-laeuft-wasser-in-die-asse/, zuletzt geprüft am24.05.2019.

Bundesgesellschaft für Endlagerung mbH (2020): Anlage IB (zum Fachbericht Teilgebiete und Anwendung Geowissenschaftliche Abwägungskriterien gemäß $\$ 24$ StandAG). Ergebnisse der Bewertung: Teil B (Keine Teilgebiete). Peine, 143

Bündnis 90/Die Grünen im niedersächsischen Landtag (2012): Abschlussbericht Parlamentarischer Untersuchungsausschuss zum Atommülllager Asse II.

CDM Smith Consult GmbH (2015): Beurteilung der gebirgsmechanischen Gefährdungsanalyse des IfG der Abbaue 3 und 4 auf der 490-m-Sohle und Abbau 2 auf der 511m-Sohle. Bochum.

Deutscher Bundestag (1959): Atomgesetz in der Fassung der Bekanntmachung vom 15. Juli 1985 (BGBl. I S. 1565), das zuletzt durch Artikel 239 der Verordnung vom 19. Juni 2020 (BGBl. I S. 1328) geändert worden ist. AtG.

Deutscher Bundestag (2013): Gesetz zur Beschleunigung der Rückholung radioaktiver Abfälle und der Stilllegung der Schachtanlage Asse II.

Deutscher Bundestag (2017): Standortauswahlgesetz vom 5. Mai 2017 (BGBl. I S. 1074), das zuletzt durch Artikel 2 Absatz 16 des Gesetzes vom 20. Juli 2017 (BGBl. I S. 2808) geändert worden ist. StandAG.

Drögemüller, Cord (2018): Schlüsselakteure der Endlager-Governance. Wiesbaden: Springer Fachmedien Wiesbaden.

Feindt, Peter H./Saretzki, Thomas (Hg.) (2010): Umwelt- und Technikkonflikte. Wiesbaden: VS Verlag für Sozialwissenschaften.

Feindt, Peter Henning (Hg.) (2005): Partizipation, Öffentlichkeitsbeteiligung, Nachhaltigkeit. Perspektiven der politischen Ökonomie. Marburg: Metropolis.

Feindt, Peter, H./Newig, Jens (2005): Politische Ökonomie von Partizipation und Öffentlichkeitsbeteiligung im Nachhaltigkeitskontext. Probleme und Forschungsperspektiven. In: Feindt, Peter Henning (Hg.): Partizipation, Öffentlichkeitsbeteiligung, Nachhaltigkeit. Perspektiven der politischen Ökonomie. Marburg: Metropolis, 9-40.

Hedin, Allan/Johansson, Adam Johannes/Lilja, Christina/Boman, Mats/Berastegui, Pedro/Berger, Rolf/Ottosson, Mikael (2018): Corrosion of copper in pure O2-free water? In: Corrosion Science 137: 1-12.

Hill, Hermann/Schliesky, Utz (Hg.) (2016): Management von Unsicherheit und Nichtwissen. 1. Auflage. Baden-Baden: Nomos Verlagsgesellschaft mbH \& Co. KG.

Hitzler, Ronald/Honer, Anne/Maeder, Christoph (Hg.) (1994): Expertenwissen: Die institutionalisierte Kompetenz zur Konstruktion von Wirklichkeit. Wiesbaden: Vieweg+Teubner Verlag. 
Hoffmann, Gregor Paul (Hg.) (2017): Organisationale Resilienz. Kernressource moderner Organisationen. Berlin, Heidelberg: Springer.

Hogrebe, Wolfram (Hg.) (2015): Grenzen und Grenzüberschreitungen. Walter de Gruyter GmbH \& Co KG.

Hultquist, G. (1986): Hydrogen evolution in corrosion of copper in pure water. In: Corrosion Science 26 (2): 173-177.

Hultquist, G./Szakálos, P./Graham, M. J./Belonoshko, A. B./Sproule, G. I./Gråsjö, L./Dorogokupets, P./Danilov, B./AAstrup, T./Wikmark, G./Chuah, G.-K./Eriksson, J.-C./Rosengren, A. (2009): Water Corrodes Copper. In: Catalysis Letters 132 (3-4): 311316.

Jürgens, Hans-Helge (1979): Atommülldeponie Salzbergwerk Asse II: Gefährdung der Biosphäre durch mangelnde Standsicherheit und das Ersaufen des Grubengebäudes.-56 S. Braunschweig.

Kärnavfallsrådet (2009): Mechanisms of Copper Corrosion in Aqueous Environments.

Kärnavfallsrådet (2010): Nuclear waste state-of-the-art report 2010. Challenges for the final repository programme: The Swedish National Council for Nuclear Waste report. Stockholm: Kärnavfallsrådet: Fritze [distributor].

Kasperson, Roger E./Renn, Ortwin/Slovic, Paul/Brown, Halina S./Emel, Jacque/Goble, Robert/Kasperson, Jeanne X./Ratick, Samuel (1988): The Social Amplification of Risk: A Conceptual Framework. In: Risk Analysis 8 (2): 177-187.

Kommission Lagerung hoch radioaktiver Abfallstoffe (2016a): Abschlussbericht: Verantwortung für die Zukunft. Ein faires und transparentes Verfahren für die Auswahl eines nationalen Endlagerstandortes. Drucksache 18/9100. Berlin.

Kommission Lagerung hoch radioaktiver Abfallstoffe (Hg.) (2016b): Beratungsunterlage zu TOP 3 der 12. Sitzung am 23. Mai 2016. Entwurf des Abschnitts B 4.2.4 NEU »Erkundungsbergwerk Gorleben«.

Kuhn, Thomas (1970): The structure of scientific revolutions. 2. Auflage. S.l.: University of Chicago Press.

Kühn, K./Klarr, K./Borchert, H. (1967): Studie über die bisherigen Laugenzuflüsse auf den Asse-Schächten und die Gefahr eines Wasser- oder Laugeneinbruchs in das Grubengebäude des Schachtes II. Herausgegeben von GSF - Gesellschaft für Strahlenforschung $\mathrm{mbH}$ München und Institut für Tieflagerung Clausthal-Zellerfeld.

Lewinski, Kai von (2016): Resilienz der Verwaltung in Unsicherheits- und Risikosituationen. In: Hill, Hermann/Schliesky, Utz (Hg.): Management von Unsicherheit und Nichtwissen. 1. Auflage. Baden-Baden: Nomos Verlagsgesellschaft mbH \& Co. KG, 239-252.

Metz, Volker (2016): Zur Freisetzung radioaktiver Isotope und dem Rückhaltepotential in den Einlagerungskammern der Schachtanlage Asse II. In: Hocke, Peter; Bechthold, Elske; Kuppler, Sophie (Hg.): Rückholung der Nuklearabfälle aus dem früheren Forschungsbergwerk Asse II. Dokumentation einer Vortragsreihe am Institut für Technikfolgenabschätzung und Systemanalyse (ITAS). Karlsruhe.

Nennen, Heinz-Ulrich/Garbe, Detlef (Hg.) (2012): Das Expertendilemma. Zur Rolle wissenschaftlicher Gutachter in der öffentlichen Meinungsbildung. Berlin: Springer.

Newig, Jens/Jahn, Stephanie/Lang, Daniel J./Kahle, Judith/Bergmann, Matthias (2019): Linking modes of research to their scientific and societal outcomes. Evidence from 
81 sustainability-oriented research projects. In: Environmental Science \& Policy 101: 147155.

NLfB (1964): Gutachten über mögliche Folgeerscheinungen der Stillegung der Schachtanlage Asse.

Popper, Karl R. (2009): Vermutungen und Widerlegungen. Das Wachstum der wissenschaftlichen Erkenntnis. In:

Schüssler, Rudolf (2015): Rationalität und Expertenstreit. In: Hogrebe, Wolfram (Hg.): Grenzen und Grenzüberschreitungen. Walter de Gruyter GmbH \& Co KG, 314-330.

Seidl, Roman/Flüeler, Thomas/Krütli, Pius/Moser, Corinne/Stauffacher, Michael (2017): Radioaktive Abfälle und Sonderabfälle im Vergleich. Zürich: Zürich, E. T.H.

Semmler (1965): Gutachten über die Gefährdungsmöglichkeit der Trinkwasserversorgung der Gemeinden in der Umgebung des Kali-Bergwerkes Asse II durch Einlagerung von radioaktiven Abfällen in den aufgelassenen Grubenbauen. Bochum.

Siegrist, Michael (2001): Die Bedeutung von Vertrauen bei der Wahrnehmung und Bewertung von Risiken. Arbeitsbericht. Stuttgart: Universität Stuttgart.

Smeddinck, Ulrich/Mintzlaff, Volker/Pönitz, Erik (2020): Entsorgungsforschung am Wendepunkt? Transdisziplinarität als Perspektive für die Forschung zur Entsorgung hochradioaktiver Abfälle - ein Projekt-Buch. 1. Auflage. Berlin: Berliner Wissenschafts-Verlag.

Strömberg, Bo/Sonnerfelt, Lena/Öberg, Henrik (2019): Exploratory what-if analysis of some debated canister failure modes in the review of a licence application for the construction and operation of a spent nuclear fuel repository in Sweden. In:Advances in Geosciences 49: 67-75.

Taleb, Nassim Nicholas (2013): Antifragilität: Anleitung für eine Welt, die wir nicht verstehen. München: Albrecht Knaus Verlag. 
